\title{
Intravitreal Ranibizumab for Diabetic Macular Edema Patients with Good Baseline Visual Acuity: A Real-Life Study
}

\author{
Abdullah Ozkaya, Mehmet Ozveren, Zeynep Alkin \\ University of Health Sciences Beyoglu Eye Training and Research Hospital
}

\begin{abstract}
Objectives: This is a report of I2-month real-life outcomes of ranibizumab treatment in diabetic macular edema (DME) patients who had a baseline visual acuity (VA) $\geq 0.6$ in decimals.

Methods: Newly diagnosed treatment-naïve DME patients with a VA $\geq 0.6$ who completed a follow-up of 12 months after treatment with ranibizumab were included retrospectively. The patients were evaluated in terms of change in best corrected visual acuity (BCVA) and central retinal thickness (CRT), and the total number of visits and injections.

Results: A total of 27 eyes of 24 patients were included. Mean BCVA at baseline, month 3, 6, 9, and I 2 was $0.77 \pm 0.11$ in decimals, $0.72 \pm 0.23,0.72 \pm 0.18,0.70 \pm 0.2 \mathrm{I}$, and $0.7 \mathrm{I} \pm 0.19$, respectively. Mean CRT at baseline, month $3,6,9$, and I 2 was $420 \pm 79,394 \pm 97,341 \pm 54,360 \pm 102$, and $350 \pm 106$ microns, respectively. The mean number of visits and injections was $4.8 \pm 1.0$ and $3.5 \pm 1.5$, respectively.

Conclusion: Ranibizumab seems to be effective in maintaining VA and improving anatomy in DME patients with a good baseline VA. However, adherence to the needed treatment regimen seems to be insufficient in real life and probably limits the visual success in this group of patients.
\end{abstract}

Keywords: Diabetic macular edema, intravitreal injection, ranibizumab, visual acuity.

\section{Introduction}

Diabetic macular edema (DME) is the most important cause of vision loss among diabetic retinopathy patient (I5). Various treatment options have been used in DME (2-5). Currently, intravitreal injection of anti-vascular endothelial growth factors (anti-VEGF), and sometimes steroids, are the preferred treatment modalities (3-5). Ranibizumab has been proven to be effective in various DME treatment regimens that were monthly, pro re nata (PRN), treat and extend, etc. (4-10). In pivotal multicenter studies, it has been demonstrated that in the first 12 months of treatment, a mean of 8 to 9 ranibizumab injections was required; however, the mean injection number dramatically decreased after the first year (4-8). Usually, it is not possible to follow the strict follow-up and retreatment criteria of these studies in real life $(9,10)$. Additionally, only patients with a visual acuity (VA) between $20 / 32-20 / 40$ and $20 / 320$ were included in these key studies, and it is unclear what the treatment outcomes are in patients with a VA better than 0.6 in decimals who require intravitreal anti-VEGF therapy (4-8). Therefore, the aim of this study was to evaluate the efficacy of intravitreal ranibizumab (IVR) in a PRN treatment regimen in DME patients with a VA of $\geq 0.6$ in decimals. 


\section{Methods}

In this retrospective, case-control study, the medical records of patients with DME who underwent IVR treatment between January 2013 and December 2015 were analyzed. Newly diagnosed treatment-naïve DME patients with a best corrected visual acuity (BCVA) $\geq 0.6$ in decimals, and who completed a treatment period of 12 months with ranibizum$a b$ in the clinic were included. Patients with a history of any other treatment for DME at admission, or who were lost to follow-up, or who received any other treatment for DME, including focal or grid laser photocoagulation in the first 12 months during our follow-up, were not included. Written, informed consent was obtained from all patients before treatment. The study adhered to the tenets of the Declaration of Helsinki.

Data collected from patient records comprised age, gender, and BCVA, central retinal thickness (CRT), and intraocular pressure (IOP) at baseline, and at months 3, 6, 9, and 12. The number of visits and injections during the first 12 months was also recorded.

All patients underwent a standard examination, including measurement of BCVA via a projection chart at 4 meters, slit-lamp biomicroscopy, measurement of IOP via applanation tonometry, and biomicroscopic fundus examination. Fundus photography, fluorescein angiography (FA) (HRA-2; Heidelberg Engineering $\mathrm{GmbH}$, Heidelberg, Germany), and optical coherence tomography (OCT) imaging (Spectralis; Heidelberg Engineering $\mathrm{GmbH}$, Heidelberg, Germany) were performed before treatment. All examinations were repeated monthly, except FA, which was performed at the physician's discretion. OCT was used to detect macular edema and for the measurement of CRT. CRT, defined as the mean thickness of the neurosensory retina in a central I-mm diameter area, was computed using OCT mapping software that accompanied the device. DME was diagnosed via FA and OCT; patients with a CRT of $>300$ microns were considered to have DME. The severity of diabetic retinopathy, the angiographic classification of DME, and the ischemic status of the macula were not assessed.

All injections were performed under sterile conditions after the application of topical anesthesia, the use of $10 \%$ povidone-iodine (Betadine; Purdue Pharma LP, Stamford, CT, USA) scrub was used on the lids and lashes, and $5 \%$ povidone-iodine was administered to the conjunctival sac. Intravitreal ranibizumab $0.5 \mathrm{mg} / 0.05 \mathrm{ml}$ (Lucentis; Novartis International AG, Basel, Switzerland) was injected through the pars plana at $3.5 \mathrm{~mm}$ posterior to the limbus with a 30-gauge needle. Patients were instructed to return to the hospital if they experienced decreased vision, eye pain, or any new symptoms.

All of the patients were to receive a loading dose of 3 consecutive monthly injections. The patients were then to be followed up monthly, and a single injection of IVR was repeated when VA decreased by I or more Early Treatment Diabetic Retinopathy Study lines, or an increase of $>100 \mathrm{mi}-$ crons in CRT on OCT images compared with the previous visit. Planned and completed visit and injection percentages were calculated to achieve a parameter for the patients' adherence to the scheduled visits and injections. The completed number of visits and injections was divided by the planned visits and injections, and then multiplied by 100 to achieve the percentages.

The primary outcome measures of this study included the change in BCVA and CRT. Secondary outcome measure was the number of visits and injections.

\section{Statistical analysis}

VA was converted to the logarithm of the minimum angle of resolution (LogMAR) for statistical analysis. Categorical variables were presented as numbers and percentages, while numerical variables were expressed as mean and standard deviation. First, the data were analyzed in terms of normality using the Shapiro-Wilk test. Since the distribution of the data was found to be normal, the VA and CRT values between the baseline and other time points were assessed with repeated measures test. Categorical variables were compared using a chi-square test. A p value $<0.05$ was considered statistically significant.

\section{Results}

A total of 27 eyes of 24 patients were included. The mean age of the patients was $54.6 \pm 8.9$ years (range: $26-67$ years); $7 \mathrm{pa}-$ tients $(25.9 \%)$ were female, and 20 patients $(74.4 \%)$ were male. The mean BCVA at baseline was $0.77 \pm 0.11$ in decimals

Table I. The mean best corrected visual acuity and central retinal thickness level at different time points during the study period

\begin{tabular}{lccccc} 
& Baseline & Month 3 & Month 6 & Month 9 & Month I2 \\
\hline BCVA, decimals (LogMAR) & $0.77 \pm 0.11$ & $0.72 \pm 0.23$ & $0.72 \pm 0.18$ & $0.70 \pm 0.21$ & $0.71 \pm 0.19$ \\
& $(0.12 \pm 0.06)$ & $(0.19 \pm 0.25)$ & $(0.20 \pm 0.15)$ & $(0.20 \pm 0.16)$ & $(0.17 \pm 0.18)$ \\
CRT, micrometers & $420 \pm 79$ & $394 \pm 97$ & $34 I \pm 54$ & $360 \pm 102$ & $350 \pm 106$ \\
\hline
\end{tabular}

BCVA: best corrected visual acuity; CRT: central retinal thickness; LogMAR: logarithm of the minimum angle of resolution. 


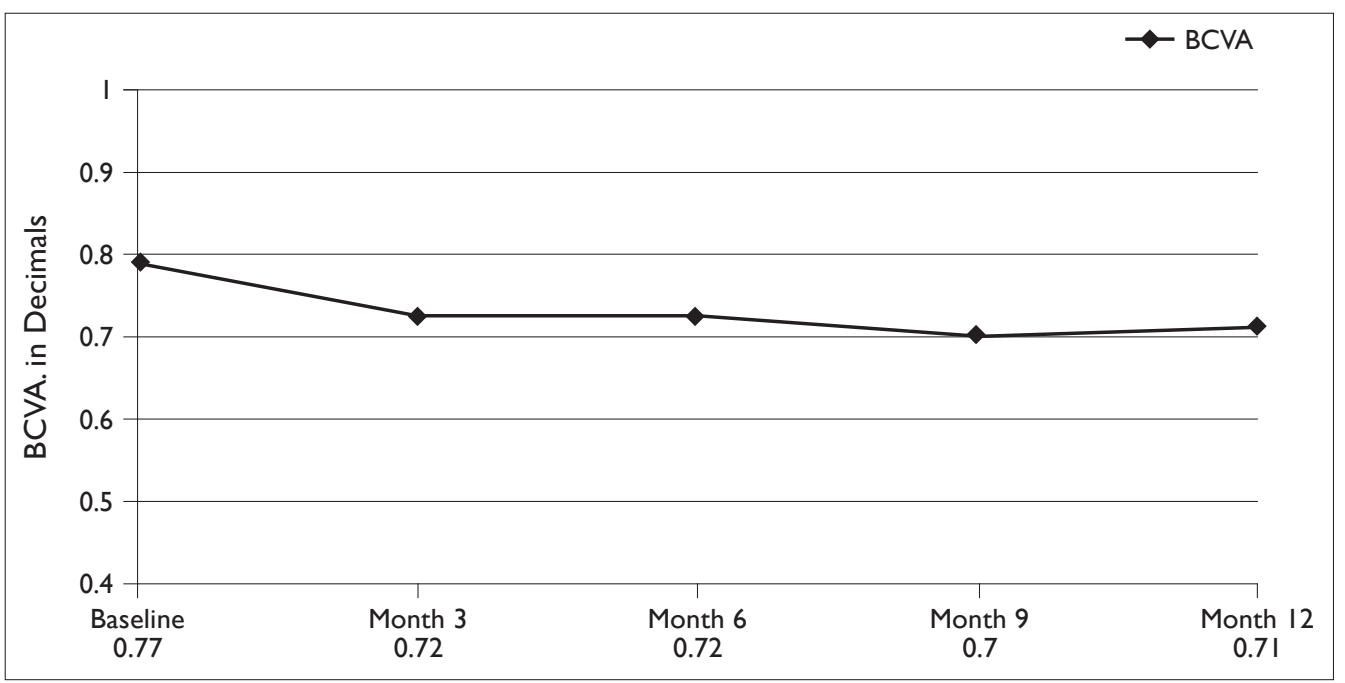

Figure I. The change in mean best corrected visual acuity at different time points.

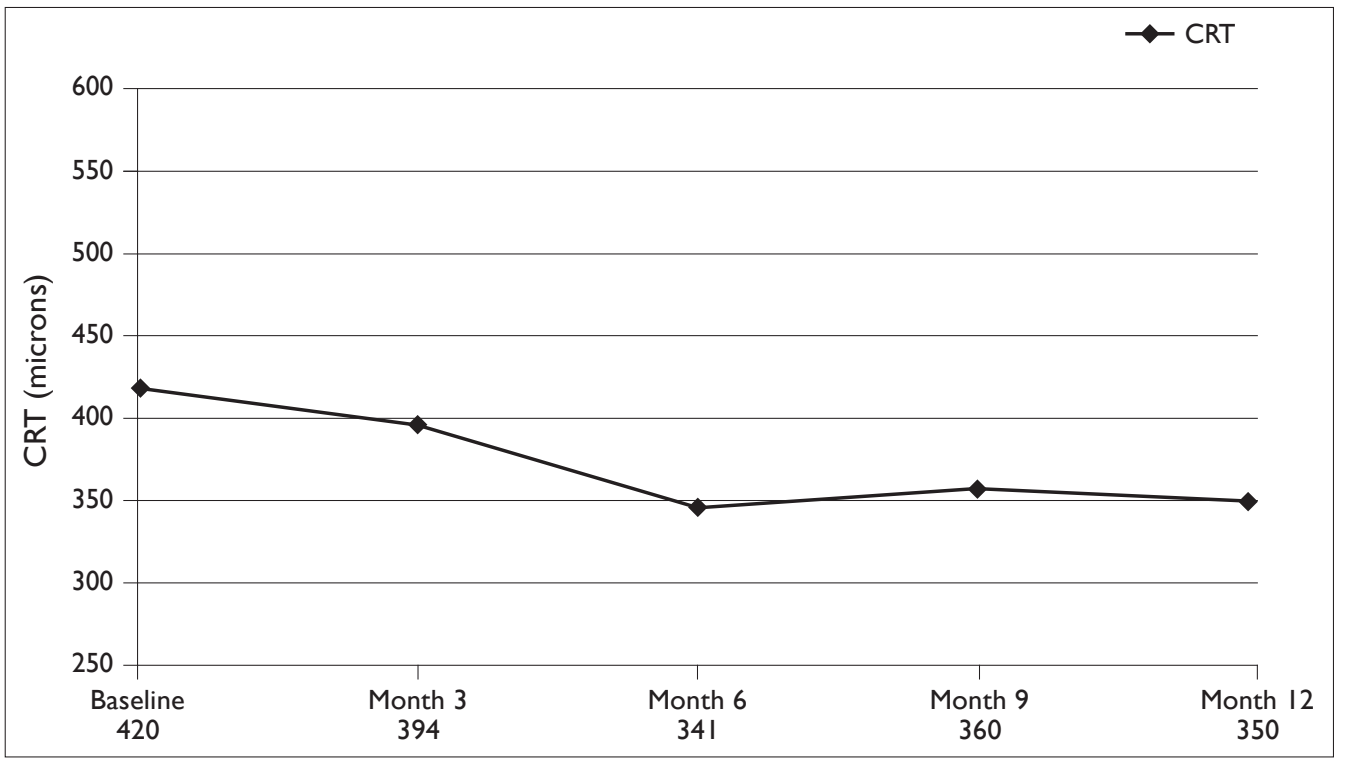

Figure 2. The change in mean central retinal thickness at different time points.

(range: $0.6-1.0$ ). The BCVA at month $3,6,9,12$ was $0.72 \pm 0.23$ (range: 0.I-I.0; $p=0.3$ ), $0.72 \pm 0.18$ (range: 0.3-I.0; $p=0.07$ ), $0.70 \pm 0.21$ (range: $0.3-1.0 ; p=0.07$ ), and $0.71 \pm 0.19$ (range: $0.2-1.0 ; p=0.3$ ), respectively (Table I) (Figure I). Three eyes (II.I\%) had visual loss of $\geq 3$ lines, and 24 eyes $(88.9 \%$ ) had stable or improved vision (loss of $<3$ lines, remained stable, or gained $\geq I$ lines) at month 12 .

The mean CRT at baseline was $420 \pm 79$ microns (range: 323-633 microns). The CRT at month 3, 6, 9, 12 was 394 \pm 97 microns (range: $256-624$ microns; $p=0.2$ ), $341 \pm 54$ microns

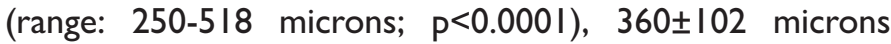
(range: $236-650$ microns; $p=0.02$ ), and $350 \pm 106$ microns (range: 252-669 microns; $\mathrm{p}=0.0 \mathrm{I}$ ), respectively (Table I) (Figure 2). At month 12, 17 of the 27 eyes (63.0\%) had a CRT of $<350$ microns.
The mean planned number of visits at month 12 was $4.9 \pm \mathrm{I} . \mathrm{I}$ (range: $3-8$ ), and the number of completed visits was $4.8 \pm 1.0$ (range: $3-8 ; 98.3 \%$ completion). The mean number of planned injections at month 12 was $4.0 \pm 1.6$ (range: $\mathrm{I}-8)$, and the number of performed injections was $3.5 \pm 1.5$ (range: I-7; 87.I\% completion). Twenty-six patients (57.8\%) received a loading dose of 3 consecutive monthly injections. No injection-related endophthalmitis was noted after a total of 95 injections.

\section{Discussion}

In this study, we evaluated the treatment outcomes and the visit and injection numbers of ranibizumab-treated DME patients with a good baseline VA. As our study was a real-life view, the visit and injection numbers were very low in com- 
parison with the prospective studies in which ranibizumab was used to treat DME patients with a VA<20/32 (approximately $<0.6$ in decimals) (4-8). The mean VA of the study population decreased from 0.77 to $0.7 \mathrm{I}$ in decimals ( 0.5 line in LogMAR; Table I), without statistical significance. The anatomical outcomes improved during the treatment course, with the mean CRT decreasing by 70 microns from 420 to 350 microns, and the difference in CRT was statistically significant at months 6, 9, and I2, compared with baseline.

In previous studies it has been reported that laser photocoagulation might be preferred in the treatment of DME patients with non-central macular edema and a BCVA of $>0.8$ in decimals (II). In our study representing daily clinical practice, we treated central-involved DME patients with intravitreal anti-VEGF injections regardless of BCVA level or angiographic type of DME. Furthermore, none of the patients included in this study underwent focal or grid laser photocoagulation therapy. Although intravitreal injections bear the risk of ocular or systemic complications, there were few complications reported (4-I0). Macular laser photocoagulation has been reported to have vision-threatening complications (12-14). Progressive enlargement of laser scars, secondary choroidal neovascular membrane formation, and reduction in the sensitivity of the central visual field are important complications of macular photocoagulation that have been reported in the literature. Schatz et al. (12) reported that II of 203 eyes with DME treated with grid laser photocoagulation showed enlargement of laser scars that progressed to the central fovea. Striph et al. (14) evaluated patients who underwent grid laser photocoagulation for DME with respect to effects on central visual field. It was reported that the threshold sensitivity of the central 5 degrees dropped by 3.44 decibels after the first treatment, and 6.86 decibels after the second. Formation of choroidal neovascularization is a common complication of macular laser treatments. The use of macular photocoagulation has become questionable since observing the promising outcomes of the use of anti-VEGF agents in visual and anatomical outcomes in the treatment of DME and their effectiveness in slowing the progression of diabetic retinopathy (4-9). In particular, the risk of enlarging scars might be an important disadvantage in the subgroup of patients with DME but a better VA.

Most previous studies did not evaluate DME patients with a good baseline VA. In most clinical studies, the upper cut-off value has been set between 20/40 and 20/32. Only in the Protocol T study were the outcomes of the subgroup of DME patients with a baseline VA between 20/40 and 20/32 evaluated (I7). The VA level was found to have increased by 8.6 letters with ranibizumab treatment at month 24 . This was a controlled, prospective study with 9 injections during the first year and 5 during the second year and the follow-up visits were performed according to strict protocols that are difficult to apply in real-world practice. Although our study results included significant visual loss, VA was preserved in nearly $90 \%$ of our patients with only 3.5 injections at month 12. Although this was not a triumphal outcome, these data might serve as a starting point to improve the follow-up visit and injection numbers in clinical practice.

The relatively small number of patients and retrospective design are the main limitations of this study. Also, neither the angiographic type of DME nor the staging of diabetic retinopathy was assessed. This was a pilot study regarding ranibizumab monotherapy in DME patients with a good baseline VA and documented real-life data of ranibizumab treatment in our clinic.

In conclusion, ranibizumab seemed to be effective at maintaining VA and improving anatomy in DME patients with a good baseline VA. However, the visual outcomes were limited by the low number of visits and injections according to the needed treatment regimen that are observed in real life.

Acknowledgements: The authors thank Dr. Hande Mefkure Ozkaya for her review of the manuscript for English editing.

\section{Disclosures}

Peer-review: Externally peer-reviewed.

Conflict of Interest: None declared.

Authorship Contributions: Involved in design and conduct of the study (AO, MO, ZA); preparation and review of the study (AO, $\mathrm{ZA})$; data collection (MO); and statistical analysis (AO).

\section{References}

I. Jusufbegovic D, Mugavin MO, Schaal S. Evolution of controlling diabetic retinopathy: Changing Trends in the Management of Diabetic Macular Edema at a Single Institution Over the Past Decade. Retina 2015;35:929-34. [CrossRef]

2. Photocoagulation for diabetic macular edema: Early Treatment Diabetic Retinopathy Study Report no. 4. The Early Treatment Diabetic Retinopathy Study Research Group. Int Ophthalmol Clin 1987;27:265-72. [CrossRef]

3. Ozkaya A, Alagoz C, Alagoz N, Gunes H, Yilmaz I, Perente I, et al. Dexamethasone implant in pseudophakic and nonglaucomatous subgroup of diabetic macular edema patients: a real life experience. Eur J Ophthalmol 2016;26:35I-5. [CrossRef]

4. Diabetic Retinopathy Clinical Research Network, Elman MJ, Aiello LP, Beck RW, Bressler NM, Bressler SB, Edwards AR, et al. Randomized trial evaluating ranibizumab plus prompt or deferred laser or triamcinolone plus prompt laser for diabetic macular edema. Ophthalmology 2010; I 17:1064-77.e35. [CrossRef]

5. Diabetic Retinopathy Clinical Research Network. A randomized trial comparing intravitreal triamcinolone acetonide and 
focal/grid photocoagulation for diabetic macular edema. Ophthalmology 2008; I I 5: I447-9. [CrossRef]

6. Mitchell P, Bandello F, Schmidt-Erfurth U, Lang GE, Massin P, Schlingemann RO, et al; RESTORE study group. The RESTORE study: ranibizumab monotherapy or combined with laser versus laser monotherapy for diabetic macular edema. Ophthalmology 201 I; I 18:6 I5-25. [CrossRef]

7. Nguyen QD, Brown DM, Marcus DM, Boyer DS, Patel S, Feiner L, et al; RISE and RIDE Research Group. Ranibizumab for diabetic macular edema: results from 2 phase III randomized trials: RISE and RIDE. Ophthalmology 20I2; I 19:789-80I. [CrossRef]

8. Payne JF, Wykoff CC, Clark WL, Bruce BB, Boyer DS, Brown DM; TREX-DME Study Group. Randomized Trial of Treat and Extend Ranibizumab with and without Navigated Laser for Diabetic Macular Edema: TREX-DME I Year Outcomes. Ophthalmology 20I7; I24:74-8I. [CrossRef]

9. Menchini U, Bandello F, De Angelis V, Ricci F, Bonavia L, Viola F, et al. Ranibizumab for Visual Impairment due to Diabetic Macular Edema: Real-World Evidence in the Italian Population (PRIDE Study). J Ophthalmol 20I5;20 I5:32484I. [CrossRef]

10. Ghanchi F, Hazel CA. South Asian diabetic macular oedema treated with ranibizumab (ADMOR)-real-life experience. Eye (Lond) 2016;30:133-8. [CrossRef]

II. Mitchell P, Wong TY; Diabetic Macular Edema Treatment Guideline Working Group. Management paradigms for diabetic macular edema. Am J Ophthalmol 2014;157:505-13. [CrossRef]
12. Schatz H, Madeira D, McDonald HR, Johnson RN. Progressive enlargement of laser scars following grid laser photocoagulation for diffuse diabetic macular edema. Arch Ophthalmol 199|;109:|549-51. [CrossRef]

13. Lewis H, Schachat AP, Haimann MH, Haller JA, Quinlan P, von Fricken $M A$, et al. Choroidal neovascularization after laser photocoagulation for diabetic macular edema. Ophthalmology 1990;97:503-10. [CrossRef]

14. Striph GG, Hart WM Jr, Olk RJ. Modified grid laser photocoagulation for diabetic macular edema. The effect on the central visual field. Ophthalmology 1988;95: I673-9. [CrossRef]

I5. Beaulieu WT, Bressler NM, Melia M, Owsley C, Mein CE, Gross JG, et al. Diabetic Retinopathy Clinical Research Network. Panretinal Photocoagulation Versus Ranibizumab for Proliferative Diabetic Retinopathy: Patient-Centered Outcomes From a Randomized Clinical Trial. Am J Ophthalmol 2016;170:206-13.

16. Writing Committee for the Diabetic Retinopathy Clinical Research Network, Gross JG, Glassman AR, Jampol LM, Inusah S, Aiello LP, Antoszyk AN, et al. Panretinal Photocoagulation vs Intravitreous Ranibizumab for Proliferative Diabetic Retinopathy: A Randomized Clinical Trial. JAMA 20I5;3 I4:2I37-46.

17. Wells JA, Glassman AR, Ayala AR, Jampol LM, Bressler NM, Bressler SB, et al. Aflibercept, Bevacizumab, or Ranibizumab for Diabetic Macular Edema: Two-Year Results from a Comparative Effectiveness Randomized Clinical Trial. Ophthalmology 2016; 123:1351-9. [CrossRef] 